\title{
Электронное участие как общественно-политический дискурс: опыт изучения дискуссий о повышении пенсионного возраста
}

\author{
О.Г. Филатова ${ }^{1}$, Ю.Г. Мисников ${ }^{2}$, Д.В. Волковский ${ }^{1}$, П.Н. Беген ${ }^{2}$ \\ ${ }^{1}$ Санкт-Петербургский государственный университет, ${ }^{2}$ Университет ИТМО \\ o.filatova@spbu.ru, yuri.misnikov@gmail.com, \\ daniil.volkovskii@yandex.ru, petyabegen@mail.ru
}

\section{Аннотация}

В статье интернет-дискурс анализируется как одна из форм электронного участия в политике. Представлен опыт анализа интернет-дискуссий на актуальную в современном российском общественно-политическом дискурсе тему, связанную с законом о пенсионной реформе. Рассматриваются возможности различных методов исследования в данном контексте, выделяются преимущества метода анализа дискурса, а также методика и итоги применения машинного обучения в семантическом анализе высказываний о пенсионной реформе. В заключение представлены важные направления дальнейших исследований. Результаты проведенного научного исследования показывают, что машинное обучение является надежным и простым в использовании инструментом для анализа содержания дискуссий в интернете и понимания их предполагаемого значения в семантических терминах, что можно использовать в качестве основы будущего каркаса для интеллектуального анализа данных и процесса вычислительной идентификации и категоризации мнений.

Ключевые слова: электронное участие, интернет-дискурс, онлайн-дискуссии, пенсионная реформа, методы исследования, дискурс-анализ, сентимент-анализ, нейронные сети, машинное обучение

Библиографическая ссылка: Филатова О.Г., Мисников Ю.Г., Волковский Д.В., Беген П.Н. Электронное участие как общественно-политический дискурс: опыт изучения дискуссий о повышении пенсионного возраста // Государство и граждане в электронной среде. Выпуск 3 (Труды XXII Международной объединенной научной конференции «Интернет и современное общество», IMS-2019, Санкт-Петербург, 19 - 22 июня 2019 г. Сборник научных трудов). - СПб: Университет ИТМО, 2019. С. 102 - 115. DOI: 10.17586/2541-979X-2019-3-102-115

\section{1. Введение}

Появление и углубление механизмов электронного участия в последние несколько лет является несомненным глобальным социально-политическим трендом. Активно развиваются социальные сети, интернет-форумы, порталы петиций, различные краудсорсинговые ресурсы, которые становятся важными площадками электронного участия, позволяющими гражданам не только получать информацию, но и активно участвовать в обсуждении вопросов, связанных с жизнью государства, влияя тем самым на принятие политических решений.

В данной статье подробно анализируется одна из форм электронного участия общественно-политический дискурс в интернете. Существующие теоретические исследования актуализируют значимость обращения к данной теме, показывая, что становящиеся все более доступными интернет-технологии способны сократить разрыв 
между властью и обществом. Анализ общественно-политического дискурса в интернете, а если говорить точнее - дискуссий в интернет-пространстве - позволяет исследовать механизмы и инструменты электронного участия граждан в политике, что может сыграть важную роль в обеспечении интерактивного взаимодействия между обществом и властью $[1$, c. 52$]$.

Исследование электронного участия как общественно-политического дискурса является одним из направлений проекта РНФ «Электронное участие как фактор динамики политического процесса и процесса принятия государственных решений». Далее будут представлены промежуточные итоги исследований, проведенных в рамках данного проекта. Цель этих исследований - аргументированно показать, что онлайн-дискуссии могут рассматриваться как форма электронного участия, поскольку они диалогичны, интерактивны, достаточно цивильны, рациональны, делиберативны. В продолжение исследований, представленных в предыдущих статьях $[1,2,3]$, в данной работе затрагиваются отдельные аспекты изучения интернет-дискуссий, связанные, прежде всего, с анализом частоты размещения постов и активности пользователей на различных интернетплатформах. В четвертом разделе представлены некоторые итоги пилотного проекта по применению технологий машинного обучения для анализа содержания интернетдискуссий.

\section{2. Теоретические основы исследования электронного участия}

Представленное ниже исследование опирается, прежде всего, на концепцию «электронного участия» в рамках более широкой концепции «электронной демократии». Согласимся с мнением А. В. Чугунова, что при изучении электронного участия необходимо опираться на классические модели демократии и соотносить их с новыми социальноэкономическими реалиями [4, с. 14$]$.

Начиная с выхода известной работы А. Макинтош в 2004 году [10] исследователями из разных стран было сделано довольно много попыток концептуализировать сферу электронного участия [см., напр.: 6-11] и мы не будем останавливаться на них подробно. К тому же, в 2007, 2008, 2012, 2016 были опубликованы довольно подробные обзоры литературы по данной теме $[12,13,14,15,16]$. Укажем лишь, что к единому определению термина «электронное участие» международное научное сообщество до сих пор так и не пришло. В 2016 году С. Шерер и М. А. Виммер провели исследование, в результате которого они разработали «метамодель электронного участия» [16]. Исследование соединило в себе сравнительный анализ литературы по концептуальным моделям, структурирующим сферу электронного участия, и моделям, описывающим структуру других моделей. Сравнительный анализ показал, что различные попытки дополняют друг друга, однако единого, всеохватывающего подхода до сих пор нет. Что касается отечественного научного дискурса, то здесь можно отметить работы, посвященные вопросам электронного участия на евразийском пространстве $[17,18]$, но таких работ крайне мало и написаны они в основном участниками представленного в данной статье гранта, а также исследования тех же авторов, посвященные делиберативности интернетдискуссий.

В данной работе мы будем опираться прежде всего на документы, разработанные ООН $[19,20]$, используя определение электронного участия, предложенное ООН в 2013 году в работе «Развитие способности участия в управлении через электронное участие», где под электронным участием подразумевается процесс вовлечения граждан через информационно-коммуникационные технологии в политику, процессы принятия решений, проектирование и доставку услуг с целью сделать их партисипативными, инклюзивными и делиберативными [21]. Близкая точка зрения высказывается и А.В. Чугуновым. По его мнению, электронным участием называется «комплекс методов и инструментов, обеспечивающих электронное взаимодействие граждан и органов власти с целью учета 
мнения граждан в государственном и муниципальном управлении, при принятии политических решений, включая реагирование на обращения граждан по широкому кругу проблем» [4, с.15].

Важно отметить, что значительную роль в контексте электронного участия играет концепция делиберативности, подробно разработанная в теории коммуникативной этики Ю. Хабермаса [22] и рассматриваемая как повседневная практика политических онлайнобсуждений, которые возникают в связи с какими-то событиями и процессами в политической сфере как на локальном, национальном, так и на глобальном уровнях [9]. Делиберативность направлена на повышение гражданской активности, связанной с принятием решений по общественным вопросам, формирование характера коммуникации, ориентированной на граждан, а также на усиление роли гражданского общества и демократических отношений в социуме в целом. Наше исследование интернет-дискуссий как раз и лежит, прежде всего, в русле концепции Ю. Хабермаса, а также опирается на методику анализа дискурса, разработанную и описанную Ю.Г. Мисниковым в $\mathrm{PhD}-$ диссертации, защищенной в 2011 году в Университете Лидса [23].

В последние несколько лет для изучения процессов формирования общественного мнения через дискурсивные формы его выражения в интернет-среде начали активно применяться алгоритмы глубокого машинного обучения нейросетей. К этому направлению, например, можно отнести и исследования в такой области искусственного интеллекта, как «аргументация и компьютинг», позволяющие устанавливать логическую взаимосвязь между утверждениями, сделанными участниками дискурса, с использованными средствами убеждения других участников в «правильности» таких утверждений [24]. Все активнее развивается компьютерная лингвистика, семантические, смысловые исследования [25], широкое распространение получило применение нейронных сетей для сентимент-анализа. Понимание этих тенденций позволило нам провести пилотное исследование с применением машинного обучения для семантического анализа дискуссий на актуальную общественнополитическую тему. Об этом речь пойдет ниже.

\section{3. Методика и результаты исследования интернет-дискурса по теме пенсионной реформы}

В декабре 2017 - марте 2018 гг. были проведены два раунда исследований интернетдискуссий, касающихся планов власти осуществить пенсионную реформу (поводом для дискуссии стала публикация законопроекта о повышении пенсионного возраста). Первый раунд представлял собой дискурс-анализ, второй - пилотное исследование в области машинного обучения.

Для анализа интернет-обсуждений, касающихся пенсионной реформы, были выбраны интернет-площадки одиннадцати разных по численности населения российских городов. Согласно своду правил Минэкономразвития РФ, города подразделяются на крупнейшие, крупные, большие, средние и малые [26]. Из каждой группы мы отобрали по два города (безсистемная выборка), выявили их наиболее популярные интернет-площадки и проанализированы онлайн-дискуссии по пенсионной тематике. Анализировались дискуссии в следующих городах: Санкт-Петербург и Волгоград (крупнейшие), Калининград и Севастополь (крупные), Братск и Нальчик (большие), Белореченск и ХантыМансийск (средние), Урюпинск и Снежинск (малые); отдельное внимание в исследовании уделялось Москве. Всего было проанализировано 5173 поста.

На интернет-форумах и порталах выбранных нами городов были проанализированы обсуждения пенсионной реформы, получены данные о том, какое количество людей в процентном соотношении выступает за повышение пенсионного возраста, а какое - против. Более того, эти данные сравнивались с результатами социологического опроса на аналогичную тему, проведенного Фондом «Общественное мнение». Если говорить о 
выявленном отношении всех участников 11 онлайн-дискуссий к пенсионной реформе, то в целом 87,2\% высказываются «против» и всего 12,8\% - «за».

Позиция «против» выше среднего показателя зафиксирована в Москве, крупных, больших и малых городах. В крупнейших и средних городах показатель положительного отношения к пенсионной реформе выше среднего (см. рис. 1).

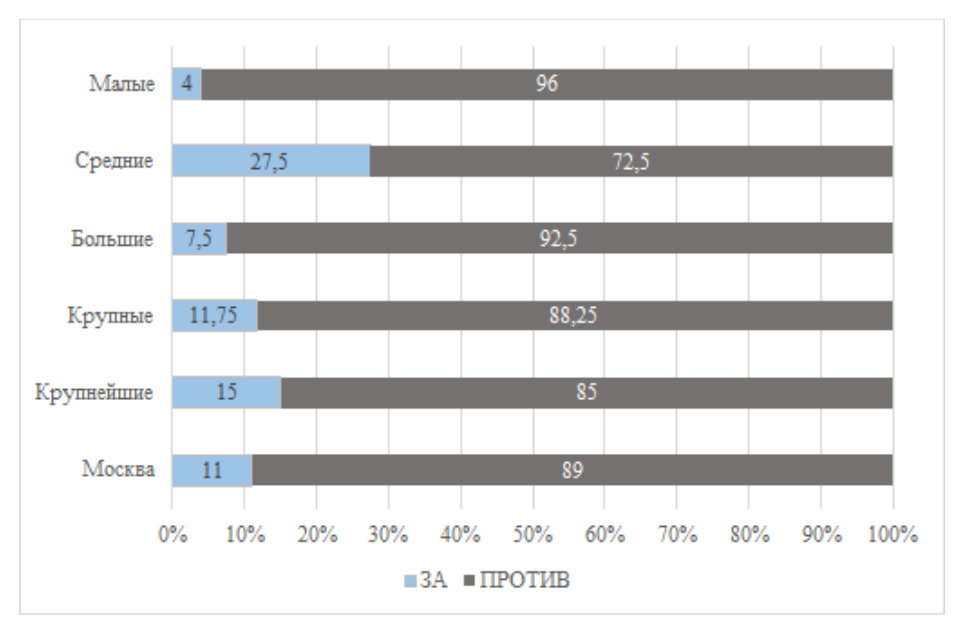

Рис. 1. Распределение позиций «за» и «против» на интернет-площадках разных по численности городов России

По результатам социологического опроса ФОМ, который проводился 26-27 мая 2018 г. в 53 субъектах РФ (1500 респондентов), общее число людей, относящихся отрицательно к идее повышения пенсионного возраста, составляет 92,3\% [27]. Этот показатель отличается от показателя, полученного нами в ходе анализа онлайн-дискуссий $(87,2 \%)$ - участники интернет-обсуждений менее отрицательно относятся к пенсионной реформе. Однако заметим, что большинство участников дискуссий оставляют по 10 и более комментариев, что в значительной степени влияет на подсчет голосов. Например, наши данные по Москве практически полностью совпали с данными ФОМ, так как участники московского форума оставляли в основном всего лишь по одному комментарию (только один участник оставил более 10 комментариев).

Сравнение с социологическими данными, полученными в тот же период, дает основание сделать одно важное заключение: граждане, участвующие в обсуждении проблемы являются более мотивированными, а, возможно, и более информированными, чем среднестатистический участник социологического опроса. Получается, что участники интернет-дискуссий демонстрируют более взвешенную позицию, чем общий негативный настрой, выявленный в результате репрезентативного социологического опроса.

Мы также исследовали активность участников дискуссий. Для нас важным было проверить, насколько соблюдается равенство участников в обсуждении и есть ли доминирующие участники дискуссии. Наша гипотеза заключалась в следующем предположении: большое число людей, принимающих участие в обсуждении вопросов на интернет-площадках, пишет относительно небольшое количество постов и небольшая часть - много сообщений. В рамках исследования частоты размещения постов было установлено, что в дискуссиях с большим числом участников (от 35) на одного пользователя приходится от 1,5 до 3 постов, что говорит о своего рода демократичности данных дискурсов, однако если количество участников меньше 35 , то количество постов, комментариев на одного человека может составлять от 8 до 15. 
В ходе исследования активности участников мы выделили пять групп: малоактивные (1 пост), умеренно активные (2-3), активные (4-5), очень активные (6-9) и гиперактивные (более 10 постов). Далее исследовалась зависимость между активностью участников и формированием их позиции в отношении пенсионной реформы, т.е.проверялась гипотеза о связи позиции «за» или «против» с количеством комментариев, оставленных интернетпользователями. Интересно, что больше всего позиций «за» зафиксировано у участников, которые выражали своё мнение либо с помощью 1 комментария (22\% от общего количества позиций «за», т.е. малоактивных), либо с помощью 10 комментариев и больше (50\% от общего количества позиций «за», т.е. гиперактивных) (см. рис. 2).

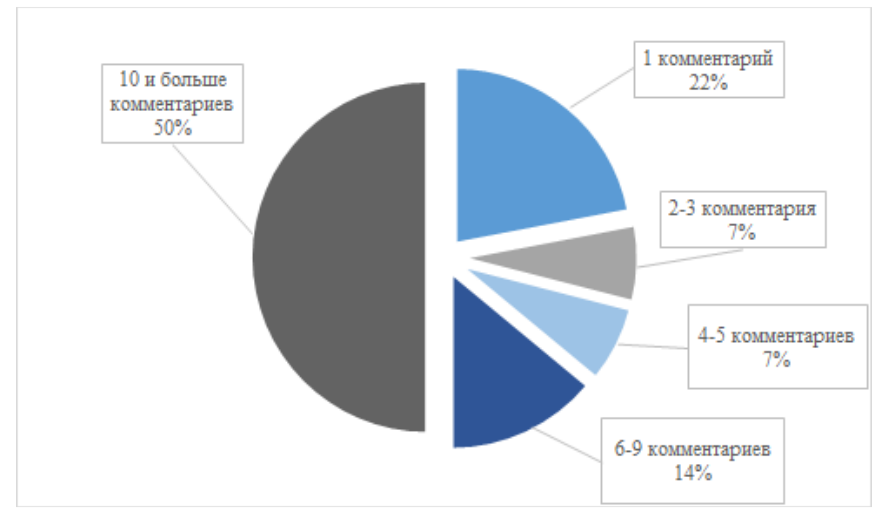

Рис. 2. Распределение позиций «За» среди участников онлайн-обсуждений

В случае выражения отрицательного отношения к пенсионной реформе ситуация отличается: в основном против реформы высказываются участники, разместившие 1 пост (15\% от общего количества позиций «против»), 6-9 (16\%) и более 10 постов (55\%) (см. рис. 3). Можно предположить, что, когда участник демонстрирует своё отношение к повышению пенсионного возраста, размещая всего один пост в дискуссии, то это говорит о том, что субъект онлайн-дискурса уверен в своей позиции и имеет чёткое представление о ней. Другое дело обстоит с теми, кто размещает 10 постов и более с позицией «против». Вероятно, эти субъекты так возмущены повышением пенсионного возраста, что готовы размещать много постов, чтобы убедить других в своей позиции. И совсем не обязательно, чтобы каждый пост был конкретно против реформы, он может касаться и других тем, но сама их активность выступает как фактор убеждения (более 3/4 всех комментариев с позицией «против» принадлежит гиперактивным участникам).

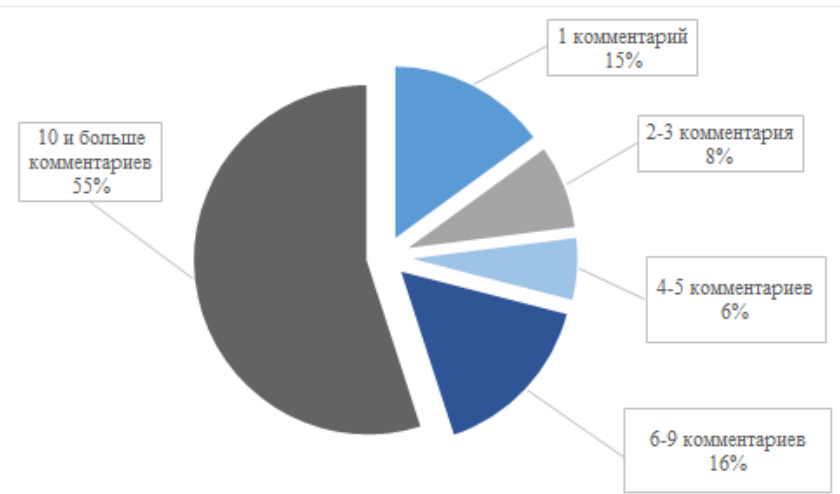

Рис. 3. Распределение позиции «Против» среди участников онлайн-обсуждений 
Другое направление исследования было связано с проверкой гипотезы об интерактивности участников онлайн-дискурса: коммуникация тех, кто пишет 10 комментариев и более, характеризуется большей интерактивностью и диалогичностью, чем коммуникация тех, кто оставляет 1 пост. Было установлено, что онлайн-обсуждения между гиперактивными пользователями (уровень интерактивности у гиперактивных участников $66,5 \%$ - см. рис. 4) почти в 3 раза более интерактивны, чем онлайн-обсуждения малоактивных (уровень интерактивности у малоактивных участников около $24 \%$ - см. рис. 4). Гиперактивные участники пишут на такие темы как пенсионная реформа, пенсионные системы и пенсии в России, СССР и заграничных странах, жизнь российских пенсионеров. К тому же, очень часто приводятся личные примеры из жизни людей, говорится о будущих негативных и позитивных последствиях принятия законопроекта, обсуждения не обходятся без многочисленных споров и оскорблений участников.

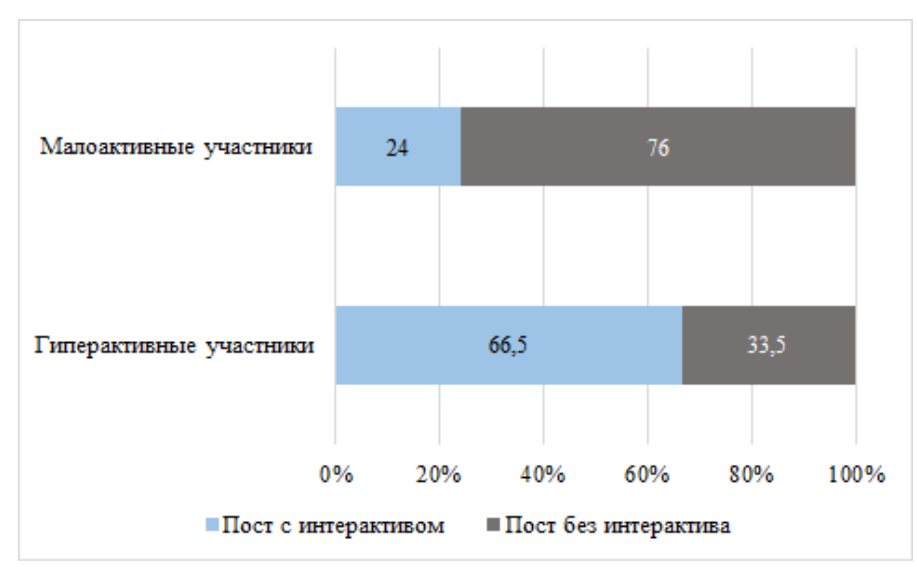

Рис. 4. Уровень интерактивности у мало/гиперактивных участников

В целом результаты исследования, конечно, еще требуют дальнейшей верификации и интерпретации. Данный этап исследования прежде всего позволил сделать вывод о том, что мнения, зафиксированные вне рамок интернет-пространства, в целом совпадают с мнениями, полученными из обсуждений на интернет-платформах. Однако анализ онлайндискурса показывает неоднозначность мнений, которые высказывались людьми по такой злободневной теме, как повышение пенсионного возраста в России. Преимущество методики дискурс-анализа по сравнению с традиционным опросом заключается в возможности выявить аргументаџию согласия или несогласия по конкретному вопросу (в данном случае по поводу пенсионной реформы). Позиция «за» или «против» может быть интуитивно ясна из текста, но дискурс-анализ позволяет обосновывать ее через механизмы согласия или несогласия с предыдущими постами, что важно в дальнейшем, в том числе при принятии политических решений.

\section{4. Методика и итоги применения машинного обучения в семантическом анализе высказываний о пенсионной реформе}

Массивы данных, использованные для дискурс-анализа, послужили основой для проведения эксперимента, связанного с глубинным машинным обучением нейросети, с тем, чтобы сеть научилась предсказывать позицию участников дискуссий по отношению к пенсионной реформе («за», «против» или «нейтрально»). Целью эксперимента было выявление возможностей применения искусственного интеллекта для более глубокого понимания результатов общественных дискуссий. 
Данные для машинного обучения (посты участников дискуссий) были представлены сначала в excel-таблицах, а потом были экспортированы в формате .csv для удобной работы с ними в программной среде. Все собранные посты (см. табл. 1) были размечены специально обученными кодировщиками ${ }^{1}$ тремя цифрами: 0 - категория «против», 1 - «за» и 2 «нейтрально».

Таблица 1. Информация о проанализированных дискуссиях

\begin{tabular}{|c|c|c|c|}
\hline \multirow{2}{*}{ № } & \multirow{2}{*}{ Город/адрес дискуссии в Интернете } & \multicolumn{2}{|c|}{ Количество } \\
\hline & & постов & участников \\
\hline 1. & $\begin{array}{l}\text { Москва } \\
\text { https://forum-msk.org/material/news/14733656.html }\end{array}$ & 126 & 85 \\
\hline 2. & $\begin{array}{l}\text { Санкт-Петербург } \\
\text { https://www.spbtalk.ru/threads/87528/ }\end{array}$ & 1793 & 42 \\
\hline 3. & $\begin{array}{l}\text { Санкт-Петербург } \\
\text { https://www.spbtalk.ru/threads/43211/ }\end{array}$ & 215 & 11 \\
\hline 4. & $\begin{array}{l}\text { Волгоград } \\
\text { https://www.forum-volgograd.ru/threads/96503/ }\end{array}$ & 284 & 67 \\
\hline 5. & $\begin{array}{l}\text { Челябинск } \\
\text { https://74.ru/forum/theme.php?id=820284 }\end{array}$ & 259 & 24 \\
\hline 6. & $\begin{array}{l}\text { Севастополь } \\
\text { https://forum.sevastopol.info/viewtopic.php?f=1\&t=1368662\&p=2 } \\
\text { 9429220\&hilit=\%D0\%BF\%D0\%B5\%D0\%BD\%D1\%81\%D0\%B8 } \\
\text { \%D0\%BE\%D0\%BD\%D0\%BD\%D1\%8B\%D0\%B9\#p29429220 }\end{array}$ & 123 & 38 \\
\hline 7. & $\begin{array}{l}\text { Калининград } \\
\text { https://www.newkaliningrad.ru/forum/topic/368296-obsuzhdenie- } \\
\text { pensionnoj-reformy/ }\end{array}$ & 561 & 23 \\
\hline 8. & $\begin{array}{l}\text { Братск } \\
\text { http://forum.bratsk.org/showthread.php?t=297973\&fbclid=IwAR18K- } \\
\text { _QjjAobA2MkG_N9i9E5baNOiuerNYjqBkxS_f-9k3AxSIK0Hu7B-Q }\end{array}$ & 120 & 35 \\
\hline 9. & $\begin{array}{l}\text { Нальчик } \\
\text { http://forum.kbrnet.ru/showthread.php?t=36248\&fbclid=IwAR2Wpw } \\
\text { VeJLgFuskoFyj6IBRzkjAv83r2pRi5EohoeAwo3SwwjkQ-f_20e3g }\end{array}$ & 683 & 27 \\
\hline 10. & $\begin{array}{l}\text { Белореченск } \\
\text { https://belorechensk.net/threads/povyshenie-vozrasta-vyxoda-na- } \\
\text { pensiju.17340/ }\end{array}$ & 178 & 22 \\
\hline 11. & $\begin{array}{l}\text { Ханты-Мансийск } \\
\text { http://86hm.ru/forum/politic/?topic_id=21469 }\end{array}$ & 80 & 13 \\
\hline 12. & $\begin{array}{l}\text { Урюпинск } \\
\text { https://forum.urup.ru/threads/povyshenie-pensionnogo-vozrasta-za-i- } \\
\text { protiv.75437/page-3?fbclid=IwAR0q3pJANjcSGhPnYVsJgNU128 } \\
\text { LmkvQVeiw2yci0HeUaLFXokw3Cc6cZ27I }\end{array}$ & 424 & 29 \\
\hline 13. & $\begin{array}{l}\text { Снежинск } \\
\text { https://forum.vega-int.ru/threads/povyshenie-pensionnogo- } \\
\text { vozrasta.113243/page-18?fbclid=IwAR1HoJMtysXAEiBgYY_tg8- } \\
\text { jYSXNcKiliH-AlqXndWGogUdaeQLDqDtpXBQ }\end{array}$ & 801 & 45 \\
\hline 14. & $\begin{array}{l}\text { KM.ru } \\
\text { http://www.km.ru/forum/economics/2018/06/18/pensionnyi-fond- } \\
\text { rf/825952-reforma-pfr-golikova-svedet-stranu-okonchatelno-s-uma }\end{array}$ & 1234 & 365 \\
\hline 15. & $\begin{array}{l}\text { Otzovik.com } \\
\text { http://otzovik.com/review_6681135.html }\end{array}$ & 269 & 70 \\
\hline 16. & $\begin{array}{l}\text { Woman.ru } \\
\text { http://www.woman.ru/health/woman-health/thread/4975755/ }\end{array}$ & 3315 & 102 \\
\hline & Всего & 10592 & 998 \\
\hline
\end{tabular}

${ }^{1}$ В качестве кодировщиков выступали студенты-магистранты СПбГУ, по три кодировщика на каждый пост для получения достоверных результатов. 
В процессе кодировки данных стало понятно, что для машинного обучения будет недостаточно того количества постов, которое использовалось для дискурс-анализа и массив данных был увеличен почти в два раза. В качестве дополнительных источников данных для машинного обучения мы обратились к трем максимально разным форумам, представляющим самые разные социальные группы: на всероссийском женском портале Woman.ru, на сайте отзывов Otzovik.com и на сайте электронного периодического издания KM.ru. Всего было закодировано 16 форумов, содержащих 10592 постов, которые разместили 998 человек (см. табл. 1).

Из всех постов: «за» - 304 сообщения (3\% от общего числа); «против» - 2510 сообщений (24\%); «нейтральное отношение» - 7778 сообщений (73\%).

Для иллюстрации того, какие комментарии оценивались в качестве «позитивного» отношения к повышению возраста, «отрицательного» или «нейтрального», приведем примеры:

«3a»;

«Всё правильно. Зарплатьл в России растут, благосостояние народа увеличивается, продолжительность жизни перевалила за 75 лет.»

«Без пенсионной реформы россияне будут стремительно беднеть. Через несколько лет их доходы сократятся на треть. Те пенсионеры, которые сейчас получают 14 тысяч, к 2026 году смогут рассчитывать лишь на 10 тысяч рублей».

«Против»:

«Реформы,должны делать мою жизнь, жизнь гражданина РФ, лучше, а не хуже. Пенсионная реформа сделает мою жизнь хуже, мне будет тяжелее жить, потому что, здоровье уже подорвано работой, в 60 лет я буду никому не нужна».

"Даже митинги не помогли! Нас не слышит никто. И никто не усльшит».

«Нейтрально»:

«Пути Бога не исповедимы. Случилось то что случилось».

«Скажите, а всем повысили или не всем? Ну там, полиции, военным тоже повысили?»

Данные .csv были загружены в программную часть с помощью библиотеки pandas². Для работы были использованы 2 столбца с данными - «текст сообщения» и «за-1/против0/нейтрально-2», загруженные как семантически связанные пары X и Y соответственно, где $\mathrm{X}$ является набором данных для обучения (обучающим примером) на входе, а $\mathrm{Y}$ - целевым результатом на выходе.

Для работы с данными в реализации алгоритма машинного обучения была произведена предварительная обработка текста с помощью встроенного класса Tokenizer библиотеки Keras, который позволяет удалить ненужные символы, привести слова к нижнему регистру, вычислить частоту встречаемости слов и т.д. Таким образом были удалены знаки пунктуации, невидимые символы, символы переноса, цифры. Не были взяты в расчет наиболее частые и наименее редкие слова, а были взяты только 3000 наиболее релевантных слова.

Далее данные были разделены на обучающую и тестовую выборки в соотношении 80/20, т.е. $20 \%$ всей совокупности данных использовались для финального тестирования обученной модели.

\footnotetext{
${ }^{2}$ Программная часть и реализация алгоритма машинного обучения была написана на языке программирования Python, версии 3.6.
} 
Обработанный текст далее был представлен в виде векторной последовательности знаков с постоянной длиной 200. Это значит, что предложение больше 200 знаков разбивалось на последовательные части. Например, в предложении «...не согласен с данной реформой...», в том месте, где на слове «согласен» встретится 200-ый знак, слово не будет разбито по частям, а перейдет полностью в следующую последовательность вместе со следующими за ним словами, а пропуски заполнятся нулями. Таким образом, частица «не» попадет в первую последовательность, а «согласен с данной реформой...»- во вторую. На рисунке 5 представлен скриншот матрицы из 200 элементов. Цифры, отличные от нуля - это некоторое слово в предложении, имеющее такой номер в словаре. 0 - это значит, что в матрице меньше 200 слов, поэтому пропуски были заполнены нулями для приведения всех матриц к единой размерности

\begin{tabular}{|c|c|c|c|c|c|c|c|c|c|c|c|c|c|}
\hline$\theta$ & $\theta$ & $\theta$ & $\theta$ & $\theta$ & $\theta$ & $\theta$ & $\theta$ & $\theta$ & $\theta$ & $\theta$ & $\theta$ & $\theta$ & $\theta$ \\
\hline$\theta$ & $\theta$ & $\theta$ & $\theta$ & $\theta$ & $\theta$ & $\theta$ & $\theta$ & $\theta$ & $\theta$ & $\theta$ & $\theta$ & $\theta$ & $e$ \\
\hline$\theta$ & $\theta$ & $\theta$ & $\theta$ & $\theta$ & $\theta$ & $\theta$ & $\theta$ & $\theta$ & $\theta$ & $\theta$ & $\theta$ & $\theta$ & $\theta$ \\
\hline$\theta$ & $\theta$ & $\theta$ & $\theta$ & $\theta$ & $\theta$ & $\theta$ & $\theta$ & $\theta$ & $\theta$ & $\theta$ & 0 & $\theta$ & $\theta$ \\
\hline$\theta$ & $\theta$ & $\theta$ & $\theta$ & $\theta$ & $\theta$ & $\theta$ & $\theta$ & $\theta$ & $\theta$ & $\theta$ & $\theta$ & $\theta$ & $\theta$ \\
\hline$\theta$ & $\theta$ & $\theta$ & $\theta$ & $\theta$ & $\theta$ & $\theta$ & $\theta$ & $\theta$ & $\theta$ & $\theta$ & 0 & $\theta$ & $\theta$ \\
\hline$\theta$ & $\theta$ & $\theta$ & $\theta$ & $\theta$ & $\theta$ & $\theta$ & $\theta$ & $\theta$ & $\theta$ & $\theta$ & $\theta$ & $\theta$ & $\theta$ \\
\hline$\theta$ & $\theta$ & $\theta$ & $\theta$ & $\theta$ & $\theta$ & $\theta$ & $\theta$ & $\theta$ & $\theta$ & $\theta$ & $\theta$ & $\theta$ & $\theta$ \\
\hline$\theta$ & $\theta$ & $\theta$ & $\theta$ & $\theta$ & $\theta$ & $\theta$ & $\theta$ & $\theta$ & $\theta$ & $\theta$ & $\theta$ & $\theta$ & $\theta$ \\
\hline$\theta$ & $\theta$ & $\theta$ & $\theta$ & $\theta$ & $\theta$ & $\theta$ & $\theta$ & $\theta$ & $\theta$ & $\theta$ & $\theta$ & $\theta$ & $\theta$ \\
\hline$\theta$ & $\theta$ & $\theta$ & $\theta$ & $\theta$ & $\theta$ & $\theta$ & $\theta$ & $\theta$ & $\theta$ & $\theta$ & $\theta$ & $\theta$ & $\theta$ \\
\hline$\theta$ & 66 & 23 & 134 & 13 & 9 & 419 & 155 & 134 & 70 & 3 & 578 & 1876 & 225 \\
\hline 2477 & 132 & 62 & 3 & 4 & 84 & 5 & 380 & 7 & 189 & 45 & 36 & 123 & 761 \\
\hline 440 & 23 & 2 & 14 & 796 & 998 & 147 & 11 & 15 & 45 & 22 & 376 & 574 & 1134 \\
\hline 913 & 499 & 60 & 11] & len & gth $=$ & 200 & & & & & & & \\
\hline
\end{tabular}

Рис. 5. Скриншот матрицы из 200 элементов

В качестве алгоритма машинного обучения была использована рекуррентная нейронная сеть с LSTM-блоками. Такой выбор связан с тем, что рекуррентные сети могут использовать свою внутреннюю память для обработки последовательностей произвольной длины, а LSTM-блоки (блоки с долго/краткосрочной памятью) являются разновидностью рекуррентных сетей и хорошо справляются с задачами классификации и прогнозирования. Такая концепция позволила нашей модели запоминать в ходе обучения предыдущие последовательности векторов для дальнейшего принятия решения и настройки весов на скрытых слоях нейронной сети.

Количество итераций, за которое модель должна была обучиться, составило 10 циклов. Для дополнительного предотвращения переобучения сети была использована функция «ранней остановки», проверяющей погрешность между функциями потерь и ошибок сети.

Для получения первых результатов мы использовали простую нейронную модель, которая состояла из следующих слоев:

- входного слоя (т.е. последовательного набора данных);

- связующего слоя, который переводит весь словарь слов к настраиваемой размерности для дальнейшего обучения;

- LSTM-блока, запоминающего информацию с предыдущих последовательностей;

- полносвязного слоя с блоком линейной ректификации, отвечающего за определение значений нейронов и их настройку;

- слоя, не допускающего переобучения сети на данных;

- выходного слоя с настраиваемым количеством выходов (в нашем случае 2 или 3).

Подчеркнем, что в рамках реализации машинного обучения было использовано два подхода для построения выходного слоя: 
1). Использование бинарной классификации (только категории «за» или «против», категория «нейтральное отношение» не использовалась), соответственно было использовано только 2814 высказываний.

2). Использование классификации по трем категориям: «за», «против» и «нейтральное отношение». Данные были использованы в максимальном объеме (10592 высказывания).

В результате применения подхода с построением бинарной классификации на выходном слое (2 категории «за» и «против») моделью были получены показатели точности примерно $89 \%$ определения. Во втором случае, при использовании трех категорий, показатели точности определения категории составили 78\%. Данные показатели точности являются достаточно хорошими для выборок с малым размером данных.

Также было проведено открытое тестирование, т.е. были взяты случайным образом данные из тестовой выборки и с помощью обученной модели были получены цифры категорий, которые сравнили с истинным результатом. Такое тестирование показало, что высказывания, относящиеся к категории «Нейтральное отношение к пенсионной реформе», определяются с наиболее высокой вероятностью (высокой точностью), чем высказывания, относящиеся к категориям «против» и «за» ввиду неравномерного распределения данных по категориям. Соответственно, для дальнейшей интерпретации результатов проведенного исследования, необходим новый эксперимент с большим количеством данных о высказываниях.

\section{5. Заключение и направления дальнейших исследований}

Исследование показало, что большинство участников интернет-дискуссий не указали четкую позицию в отношении увеличения пенсионного возраста и суждения были в целом неоднозначны. Отсюда возникает сложность принятия решений властями, которые учитывали бы разные мнения активных участников интернет-дискурса. Следует отметить, что мы исходим из оптимистической предпосылки, что создание методики дискурс-анализа и инструментария, создающего возможность выявить аргументацию и вычленить позиции дискутантов, будет востребовано лицами, участвующими в принятии решений. Неоднозначность является универсальной проблемой и одновременно даёт повод задуматься, как могут работать системы и модели поддержки принятия государственных решений с учетом мнения граждан. Тематика пенсионной реформы - это только один из возможных примеров исследования. Для исследования общественно-политического дискурса важна тема, где мнения не только поляризуются на две группы, но и где возникает множество таких групп. В свою очередь, это побуждает изучать и выявлять в текстах дискуссий утверждения, которые могут быть поддержаны или оспорены. Это, безусловно, чрезвычайно важно для осуществления грамотной государственной политики. Такие исследования необходимо продолжать, делая особый акцент на изучении уровней аргументированности, интерактивности, диалогичности, рациональности, интернетобсуждений.

Методика анализа интернет-дискуссий может и должна быть усовершенствована. Для этого необходимо задействовать современные программы и инструменты обработки данных, возможности искусственного интеллекта и нейронных сетей.

Общая концепция проведенного научного исследования перспективна в той степени, в которой его можно использовать в качестве основы будущего каркаса для интеллектуального анализа данных и процесса вычислительной идентификации и категоризации мнений. Проведенный эксперимент показывает, что машинное обучение является надежным и простым в использовании инструментом для анализа содержания дискуссий в интернете и понимания их предполагаемого значения в семантических терминах. Точность прогнозирования достигает более $80 \%$ при наборе обучающих данных, насчитывающем около 7 тыс. сообщений, однако затем заметно уменьшается, когда размер набора данных уменьшается. Исследования в данном направлении необходимо 
продолжить, чтобы предложить решения для использования искусственного интеллекта в целях более глубокого понимания результатов общественных дискуссий.

В дальнейшем планируется выявить, на какие именно элементы системы государственного управления и публичной политики общественно-политический дискурс в интернете оказывает наибольшее влияние. С прикладной точки зрения важным направлением исследований является разработка прототипа приложения по содействию участникам интернет-дискуссий в сглаживании противоречий, работающего на основе обученных нейросетей, что позволит вывести аналитику контента общественных обсуждений на качественно новый уровень.

Работа выполнена при поддержке РНФ, проект №18-18-00360 «Электронное участие как фактор динамики политического процесса и процесса принятия государственных решений».

\section{Литература}

[1] Мисников Ю. Г., Филатова О. Г., Чугунов А. В. Электронное взаимодействие власти и общества: направления и методы исследований // Научно-технические ведомости СПбГПУ. Гуманитарные и общественные науки. 2016. №1. С. 52-60.

[2] Chugunov A., Filatova O., Misnikov Y. Citizens' deliberation online as will-formation: The impact of media identity on policy discourse outcomes in Russia // IFIP International Federation for Information Processing 2016. Published by Springer International Publishing Switzerland 2016. E. Tambouris et al. (Eds.): ePart 2016, LNCS 9821. P. 67-82. DOI: 10.1007/978-3-319-45074-2_6.

[3] Misnikov Y., Filatova O., Chugunov A. Converting the outcomes of citizens discourses in the cyberspace into policy inputs for more democratic and effective government // Beyond Bureaucracy: Towards Sustainable Governance Informatisation. Springer, 2017. P. 259-287.

[4] Чугунов А. В. Электронное участие в контексте трансформации концепции демократии // Управленческое консультирование. 2017. № 8. С. 14-20. URL: https://sziu.ranepa.ru/images/nauka/UK_DOI/8_17/Chugunov_8_17.pdf.

[5] Macintosh A. Characterizing e-participation in policy-making // Proceedings of the 37th HICSS. IEEE Computer Society, Los Alamitos. 2004. URL: http://www. computer.org/csdl/proceedings/hicss/2004/2056/05/205650117a.pdf.

[6] Kalampokis E., Tambouris E., Tarabanis K.A. A domain model for eParticipation // Mellouk A., Bi J., Ortiz G., et al. (eds) Proceedings of the Third International Conference on Internet and Web Applications and Services, IEEE Computer Society, Los Alamitos. 2008. P. 25-30.

[7] Macintosh A. eParticipation in policy-making. The research and the challenges, exploiting the knowledge economy: issues, applications, case studies. Amsterdam: IOS Press, 2006.

[8] Macnamara J. 2010. The quadrivium of online public consultation: Policy, culture, resources, technology // Australian Journal of Political Science. 2010. Vol. 45 (2). P. 227-244.

[9] Medaglia R. Measuring the diffusion of eParticipation: A survey on Italian local government // Information Polity. 2007. Vol. 12 (4). P. 265-280.

[10]Prosser A. 2013. eParticipation on the European Union Level // Technology-Enabled Innovation for Democracy, Government and Governance. Springer Berlin Heidelberg, 2013. P. $1-8$.

[11]Smith S., Macintosh A., Millard J. A three-layered framework for evaluating e-participation // Int. J. Electron. Gov. 2011. Vol. 4(4). P. 304-321. DOI: 10.1504/IJEG.2011.046013.

[12]Sanford C., Rose J. Characterizing eParticipation // Int. J. Inf. Manag. 2007. Vol. 27. P. 406421.

[13]Sæbø Ø., Rose J., Flak L.S. The shape of eParticipation. Characterizing an emerging research area // Government Information Quarterly. 2008. Vol. 25(3). P. 400-428. DOI: 10.1016/j.giq.2007.04.007. 
[14]Susha I., Gronlund A. eParticipation research: Systematizing the field // Government Information Quarterly. 2012. Vol. 29. P. 373-382.

[15]Alarabiat A., Sá Soares D., Estevez E. Electronic Participation with a Special Reference to Social Media - A Literature Review // IFIP International Federation for Information Processing 2016. Published by Springer International t Publishing Switzerland 2016. E. Tambouris et al. (Eds.): ePart 2016, LNCS 9821. P. 41-52. DOI: 10.1007/978-3-319-45074-2 4.

[16]Scherer S., Wimmer M. A Metamodel for the E-Participation // IFIP International Federation for Information Processing 2016. Published by Springer International Publishing Switzerland 2016. E. Tambouris et al. (Eds.): ePart 2016, LNCS 9821. P. 3-16. DOI: 10.1007/978-3-31945074-2 1 .

[17]Bolgov R., Karachay V. E-Governance Institutions Development in the Eurasian Economic Union: Case of the Russian Federation // ACM International Conference Proceeding Series. 9th International Conference on Theory and Practice of Electronic Governance, ICEGOV Montevideo; Uruguay, 2016. DOI: 10.1145/3209415.3209435.

[18] Filatova O., Balabanova S., Golubev V., Ibragimov I. E-participation in EEU countries: a case study of government websites // eGose '17 Proceedings of the International Conference on Electronic Governance and Open Society: Challenges in Eurasia. ACM, NY, 2017. P. 145151. DOI: $10.1145 / 3129757.3129782$.

[19]E-Participation Index. URL: https://publicadministration.un.org/egovkb/enus/About/Overview/E-Participation.

[20] UNDESA's overview of e-participation models. Prepared by Nahleen Ahmed, DPADM. URL: http://unpan1.un.org/intradoc/groups/public/documents/un/unpan023622.pdf.

[21]Concept paper 'Developing capacity for participatory governance through e-participation // DPADM, 2013. URL: http://workspace.unpan.org/sites/internet/Documents/ CONCEPT\%20PAPER\%20e-Participation\%2001.30.13.pdf.

[22] Habermas J. Moral consciousness and communicative action. Cambridge: Polity Press, 1992.

[23]Misnikov Y. Public Activism Online in Russia: Citizens' Participation in Webbased Interactive Political Debate in the Context of Civil Society. Development and Transition to Democracy. University of Leeds, 2011.

[24]Snaith M., Lawrence J., Reed C. Mixed Initiative Argument in Public Deliberation. // De Cindio, F., Macintosh, A., Peraboni, C. (Eds.) From e-participation to Online Deliberation, Proceedings of the Fourth International Conference on Online Deliberation, 2010. P. 2-13.

[25]Peng Wang, Jiaming Xu, Bo Xu, Cheng-Lin Liu, Heng Zhang Fangyuan Wang, Hongwei Hao. Semantic Clustering and Convolutional Neural Network for Short Text Categorization // Proceedings of the 53rd Annual Meeting of the Association for Computational Linguistics and the 7th International Joint Conference on Natural Language Processing. Beijing, China, July 26-31, 2015. P. 352-357. URL: http://www.aclweb.org/anthology/P15-2058.

[26]СП 42.13330.2011 Градостроительство. Планировка и застройка городских и сельских поселений. Актуализированная редакция СНиП 2.07.01-89 (с поправкой). М., 2011.

[27]О пенсионном возрасте и отношении к идее его повысить // Портал ФОМ. 2018. URL: https://fom.ru/posts/14043. 


\title{
E-Participation as a Socio-Political Discourse: Case Study on Raising the Retirement Age
}

\author{
O.G. Filatova ${ }^{1}$, Y.G. Misnikov ${ }^{2}$, D.V. Volkovskii ${ }^{1}$, P.N. Begen ${ }^{2}$ \\ ${ }^{1}$ Saint-Petersburg State University ${ }^{2}$ ITMO University
}

The experience of Internet discussions analyzing on actual in the modern Russian socio-political discourse theme connecting with the law on pension reform is revealed in the article. The discussions on Internet platforms of different Russian cities are considered as a form of eparticipation in politics. The results of a scientific study show that machine learning is a reliable and easy-to-use tool for analyzing the content of discussions on the Internet and understanding their intended meaning in semantic terms, which can be used as the basis for a future framework for data mining and the process of computational identification and categorization of opinions.

Keywords: e-participation, Internet discourse, online discussions, pension reform, research methods, discourse analysis, sentiment analysis, neural networks, machine learning

Reference for citation: Filatova O.G., Misnikov Y.G., Volkovskii D.V., Begen P.N. EParticipation as a Socio-Political Discourse: Case Study on Raising the Retirement Age // The State and Citizens in the Electronic Environment. Vol. 3 (Proceedings of the XXII International Joint Scientific Conference «Internet and Modern Society», IMS-2019, St. Petersburg, June 19-22, 2019). - St. Petersburg: ITMO University, 2019. P. 102 - 115. DOI: 10.17586/2541-979X-20193-102-115

\section{Reference}

[1] Misnikov Y.G., Filatova O.G., Chugunov A.V. E-interaction of authority and society: research directions and methods // Scientific and technical statements of SPbSPU. Humanitarian and Social Studies. 2016. №1. P. 52-60.

[2] Chugunov A., Filatova O., Misnikov Y. Citizens' deliberation online as will-formation: The impact of media identity on policy discourse outcomes in Russia // IFIP International Federation for Information Processing 2016. Published by Springer International Publishing Switzerland 2016. E. Tambouris et al. (Eds.): ePart 2016, LNCS 9821. P. 67-82. DOI: 10.1007/978-3-319-45074-2_6.

[3] Misnikov Y., Filatova O., Chugunov A. Converting the outcomes of citizens discourses in the cyberspace into policy inputs for more democratic and effective government // Beyond Bureaucracy: Towards Sustainable Governance Informatisation. Springer, 2017. P. 259-287.

[4] Chugunov A.V. E-participation in context of transformation of democracy concept // Government consulting. 2017. № 8. P. 14-20.

[5] Macintosh A. Characterizing e-participation in policy-making. In: Proceedings of the 37th HICSS. IEEE Computer Society, Los Alamitos. 2004. URL: http://www. computer.org/csdl/proceedings/hicss/2004/2056/05/205650117a.pdf.

[6] Kalampokis E., Tambouris E., Tarabanis K.A. A domain model for eParticipation // Mellouk, A., Bi, J., Ortiz, G., et al. (eds) Proceedings of the Third International Conference on Internet and Web Applications and Services, IEEE Computer Society, Los Alamitos. 2008. P. 25-30.

[7] Macintosh A. eParticipation in policy-making. The research and the challenges, exploiting the knowledge economy: issues, applications, case studies. Amsterdam: IOS Press. 2006.

[8] Macnamara J. 2010. The quadrivium of online public consultation: Policy, culture, resources, technology. Australian Journal of Political Science. 2010. 45, 2, 227-244.

[9] Medaglia R. Measuring the diffusion of eParticipation: A survey on Italian local government. Information Polity. 2007. № 12 (4). P. 265-280. 
[10]Prosser A. 2013. eParticipation on the European Union Level. Technology-Enabled Innovation for Democracy, Government and Governance. Springer Berlin Heidelberg, 2013. P. 1-8.

[11]Smith S., Macintosh A., Millard J. A three-layered framework for evaluating e-participation. Int. J. Electron. Gov. 2011. №4(4), 304-321 URL: doi:10.1504/IJEG.2011.046013.

[12]Sanford C., Rose J. 2007. Characterizing eParticipation. Int. J. Inf. Manag. 27, 406-421.

[13]Sæbø Ø., Rose J., Flak L.S. The shape of eParticipation. Characterizing an emerging research area. Gov. Inf. Q. 2008. № 25(3), 400-428. URL: doi:10.1016/j.giq.2007.04.007.

[14]Susha I., Gronlund A. 2012. eParticipation research: Systematizing the field. Government Information Quarterly. 2012. № 29. P. 373-382.

[15]Alarabiat A., Sá Soares D., Estevez E. Electronic Participation with a Special Reference to Social Media - A Literature Review. IFIP International Federation for Information Processing 2016. Published by Springer International t Publishing Switzerland 2016. All Rights Reserved. E. Tambouris et al. (Eds.): ePart 2016, LNCS 9821, 41-52. URL: DOI: 10.1007/978-3-31945074-2 4.

[16]Scherer S., Wimmer M. A Metamodel for the E-Participation. In IFIP International Federation for Information Processing 2016. Published by Springer International Publishing Switzerland 2016. E. Tambouris et al. (Eds.): ePart 2016, LNCS 9821, 3-16. URL: DOI: 10.1007/978-3319-45074-2_1.

[17]Bolgov R., Karachay V. E-Governance Institutions Development in the Eurasian Economic Union: Case of the Russian Federation // ACM International Conference Proceeding Series. 9th International Conference on Theory and Practice of Electronic Governance, ICEGOV Montevideo; Uruguay. 2016.

[18] Filatova O., Balabanova S., Golubev V., Ibragimov I. E-participation in EEU countries: a case study of government websites // eGose '17 Proceedings of the Internationsl Conference on Electronic Governance and Open Society: Challenges in Eurasia. ACM, NY, 2017. P. 145151. URL: doi>10.1145/3129757.3129782.

[19]E-Participation Index. URL: https://publicadministration.un.org/ egovkb/enus/About/Overview/E-Participation.

[20] UNDESA's overview of e-participation models. Prepared by Nahleen Ahmed, DPADM. URL: http:/unpan1.un.org/intradoc/groups/public/documents/un/unpan023622.pdf

[21]Concept paper 'Developing capacity for participatory governance through e-participation // DPADM, 2013. URL: http://workspace.unpan.org/sites/internet/Documents/ CONCEPT\%20PAPER\%20e-Participation\%2001.30.13.pdf.

[22]Habermas J. Moral consciousness and communicative action. Cambridge: Polity Press, 1992.

[23]Misnikov Y. Public Activism Online in Russia: Citizens' Participation in Webbased Interactive Political Debate in the Context of Civil Society. Development and Transition to Democracy. University of Leeds, 2011.

[24]Snaith M., Lawrence J., Reed C. Mixed Initiative Argument in Public Deliberation. // De Cindio, F., Macintosh, A., Peraboni, C. Eds. From e-participation to Online Deliberation, Proceedings of the Fourth International Conference on Online Deliberation, 2010. P. 2-13.

[25]Peng Wang, Jiaming Xu, Bo Xu, Cheng-Lin Liu, Heng Zhang Fangyuan Wang, Hongwei Hao. Semantic Clustering and Convolutional Neural Network for Short Text Categorization // Proceedings of the 53rd Annual Meeting of the Association for Computational Linguistics and the 7th International Joint Conference on Natural Language Processing. Beijing, China, July 26-31, 2015. P. 352-357. URL: http://www.aclweb.org/anthology/P15-2058.

[26] SR 42.13330.2011 Urban development. Planning and building of urban and rural settlements. Updated edition 2.07.01-89 (with mark). M., 2011.

[27]About pension age and attitude towards of increasing it//Portal FPO. 2018. URL: https://fom.ru/posts/14043. 